\title{
Nucleation in Aqueous KCl Solutions Induced by Laser Pulses ${ }^{+}$
}

\author{
Andrea Taschin ${ }^{1,2}$, Paolo Bartolini ${ }^{1}$, Stefano Benci ${ }^{1}$ and Renato Torre ${ }^{1, *}$ \\ 1 European Lab. for Non-Linear Spectroscopy (LENS) and Dip. di Fisica ed Astronomia, Università di \\ Firenze, via N. Carrara 1, 50019 Sesto Fiorentino, Firenze, Italy \\ 2 ENEA, Centro Ricerche Frascati, Via E. Fermi 45, I-00044 Frascati, Roma, Italy \\ * Correspondence: torre@lens.unifi.it \\ + Presented at the 37th International Symposium on Dynamical Properties of Solids (DyProSo 2019), Ferrara, \\ Italy, 8-12 September 2019.
}

Published: 5 September 2019

The nucleation processes are still today between the more intriguing problems of the condensed matter physics; they have been described by different models, but their understanding I still far to be complete [1]. In particular, we are missing the experimental investigations of the first nucleation events due to their unpredictable localization in the time and space coordinates.

The nucleation in saturated solutions can be induced by a short laser pulse of high intensity [2]; a series of experimental studies have proved that this phenomenon occur in aqueous solutions of very different nature, where the solutes can vary from simple salts to complex biological molecules [3]. Even if the NonPhotochemical Laser Induced Nucleation (NPLIN) is known from more than 20 years, several fundamental aspects of it remain open problems [4]. Nevertheless, the NPLIN enables the possibility to perform spectroscopic measurements during the first steps of crystal nucleation and growth, opening the harvesting of new valuable information on this elusive phenomenon.

We performed an experimental investigation of the nucleation phenomena induced by a 25 picosecond infrared laser pulse in a supersaturated aqueous $\mathrm{KCl}$ solution. In particular, we developed an experimental set-up able to perform a very fast imaging (up to $5 \times 10^{5}$ frame/s) of the solution modifications induced by the laser pulse. The fast imaging shows a series of physical processes taking place in the solution between the laser pulse arrival and the show up of the crystal nucleus, see Figure 1.
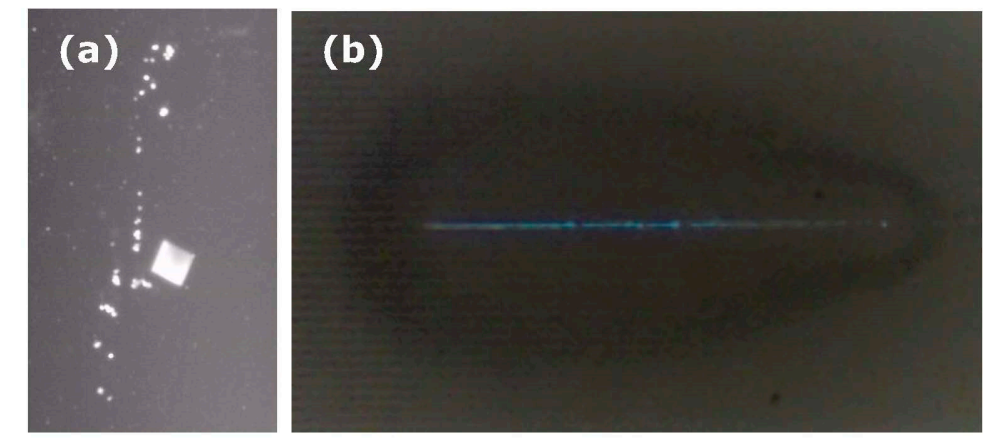

Figure 1. (a) $\mathrm{KCl}$ crystals induced by a laser pulse of 25 ps; (b) plasma line and shockwave preceding the nucleation phenomena, captured by an ultrafast camera with a $25 \mu$ s shutter time. 


\section{References}

1. Kelton, K.F.; Frenkel, D. Preface: Special Topic on Nucleation: New Concepts and Discoveries. Preface J. Chem. Phys. 2016, 145, doi:10.1063/1.4967522.

2. Garetz, B.A.; Aber, J.E.; Goddard, N.L.; Young, R.G.; Myerson, A.S. Nonphotochemical, polarizationdependent, laser-induced nucleation in supersaturated aqueous urea solutions. Phys. Rev. Lett. 1996, 77, 3475.

3. Li, W.; Ikni, A.; Scouflaire, P.; Shi, X.; El Hassan, N.; Gémeiner, P.; Gillet, J.-M.; Spasojević-de Biré, A. NonPhotochemical Laser-Induced Nucleation of Sulfathiazole in a Water/Ethanol Mixture. Cryst. Growth Des. 2016, 16, 2514.

4. Kacker, R.; Dhingra, S.; Irimia, D.; Ghatkesar, M.K.; Stankiewicz, A.; Kramer, H.J.M.; Eral, H.B. Multiparameter Investigation of Laser-Induced Nucleation of Supersaturated Aqueous KCI Solutions. Cryst. Growth Des. 2018, 18, 312.

(C) 2019 by the authors. Licensee MDPI, Basel, Switzerland. This article is an open access article distributed under the terms and conditions of the Creative Commons Attribution (CC BY) license (http://creativecommons.org/licenses/by/4.0/). 